\title{
RELATIVE OCKHAM LATTICES: THEIR ORDER-THEORETIC AND ALGEBRAIC CHARACTERISATION
}

\author{
by G. BORDALO $\dagger$ and H. A. PRIESTLEY
}

(Received 22 September, 1988)

1. Introduction. Given a variety $\mathscr{A}$ of lattice-ordered algebras, a lattice $L$ is said to be a relative $\mathscr{A}$-lattice if every closed interval $[a, b]$ of $L$ may be given the structure of an algebra in $\mathscr{A}$ (in other words, is the reduct of a member of $\mathscr{A}$-not necessarily unique). This paper discusses the characterisation in terms of forbidden substructures of finite relative $\mathscr{A}$-lattices. We treat a large class of varieties $\mathscr{A}$ of distributive-lattice-ordered algebras. For these varieties, the finite algebras can be described dually in terms of finite ordered sets, so that order-theoretic results and techniques prove valuable.

Our study was prompted by a desire to set in a wider context the following characterisations of relative de Morgan lattices and of relative Stone lattices.

TheOREM 1.1. (J. C. Varlet [24]) Let $L$ be a finite distributive lattice. Then the following are equivalent:

(1) $L$ is a relative de Morgan lattice;

(2) $L$ has no closed interval isomorphic to $1 \oplus 2^{2}$ or to $\mathbf{2}^{2} \oplus \mathbf{1}$;

(3) $L$ is a direct product of chains.

Theorem 1.2, (G. Bordalo [9]) Let $L$ be a finite distributive lattice. Then the following are equivalent:

(1) $L$ is a relative Stone lattice;

(2) L has no closed interval isomorphic to $1 \oplus \mathbf{2}^{2}$;

(3) the ordered set of join-irreducible elements of $L$ is a disjoint union of trees.

Thus when $\mathscr{A}$ is either the variety of de Morgan algebras or the variety of Stone algebras the finite relative $\mathscr{A}$-lattices have a characterisation which can be neatly expressed in terms of the poset of join-irreducible elements (condition (3) in Theorem 1.1 being equivalent to the poset of join-irreducible elements of $L$ is a disjoint union of chains). Alternatively, these lattices can be described via forbidden substructures (in each case, forbidden intervals of small cardinality) - a technique which has proved valuable in graph theory, order theory and lattice theory. More degenerately, a similar characterisation is available when $\mathscr{A}=\mathbf{B}$, the variety of Boolean algebras; see A. Björner [4] for a discussion of lattices (not necessarily distributive) with $\mathbf{3}$ as a forbidden interval.

One is naturally led to ask whether Theorems 1.1 and 1.2 are specific to the varieties to which they refer, or whether there are analogous results for other varieties of distributive-lattice-ordered algebras. A natural setting for such an investigation is the lattice of subvarieties of the variety $\mathbf{O}$ of Ockham algebras, to which Boolean algebras, de Morgan algebras and Stone algebras all belong. An Ockham algebra is an algebra $(L ; \vee$, $\wedge, 0,1)$ of type $(2,2,1,0,0)$, where $(L ; \vee, \wedge, 0,1)$ is a bounded distributive lattice and $\sim$ is a dual endomorphism. The identity $\sim^{2} a=a$ and the identities $a \wedge \sim a=0$ and $\sim a \vee \sim^{2} a=1$ define respectively the subvarieties $\mathbf{M}$ (of de Morgan algebras) and $\mathbf{S}$ (of

† The first author acknowledges with gratitude the support of the British Council and the hospitality of the Mathematical Institute, Cxford.

Glasgow Math. J. 32 (1990) 47-66. 
Stone algebras). Two other Ockham varieties play an important role in our investigations: $\mathbf{S}^{d}$ (dual Stone algebras) and $\mathbf{K}$ (Kleene algebras). The algebra $(L ; \vee, \wedge, \sim, 0,1)$ is in $\mathbf{S}^{d}$ if and only if $\left(L^{d} ; \vee, \wedge, \sim, 0,1\right) \in \mathbf{S} ; L^{d}$ denotes the order dual of $L$. The variety $\mathbf{K}$ is the subvariety of $M$ defined by the identity $a \wedge \sim a \leqslant b \vee \sim b$. It is well known that the lattice of (non-trivial) subvarieties of $\mathbf{M}$ is a 3-element chain: $\mathbf{B} \subset \mathbf{K} \subset \mathbf{M}$.

Given a variety $\mathscr{A}$ of lattice-ordered algebras, we denote its lattice of subvarieties by $\Lambda(\mathscr{A})$ and by $\Lambda_{f}(\mathscr{A})$ the subset of $\Lambda(\mathscr{A})$ consisting of those varieties which are generated by a single finite algebra.

The lattice $\Lambda(\mathbf{0})$ has a rich structure, without being intractable. Its basic structure, and relation to $\Lambda_{f}(\mathbf{0})$, was revealed by $A$. Urquhart $([22,23])$, following pioneering work by J. Berman [3] (see Section 4 for more details). Further contributions were made by M.S. Goldberg in [15] and [16]. In addition, a number of particular subvarieties close to the bottom of $\Lambda(\mathbf{0})$ have been intensively investigated, including the variety of MS-algebras studied by T. S. Blyth and J. C. Varlet et al. (see [1], [5], [6], [7] and [8] for basic facts and further references).

The complexity of $\Lambda(\mathbf{O})$ is such that the determination of the finite relative $\mathscr{A}$-algebras for every $\mathscr{A} \in \Lambda(\mathbf{O})$ is too big a project for a single paper. We therefore concern ourselves here primarily with a more restricted problem, which is most conveniently presented by considering an equivalence relation $\approx$ on $\Lambda(\mathbf{0})$. For $\mathscr{A}$, $\mathscr{B} \in \Lambda(\mathbf{0})$, define $\mathscr{A} \approx \mathscr{B}$ if and only if the class of finite relative $\mathscr{A}$-algebras coincides with the class of finite relative $\mathscr{B}$-algebras. We can then state our immediate goal as being to calculate the equivalence classes of $\mathbf{B}, \mathbf{M}, \mathbf{S}$ and $\mathbf{S}^{d}$ (or at least their intersections with $\Lambda_{f}(\mathbf{O})$ ). In Section 3 we shall give a general description of these equivalence classes. This work makes use only of the most basic facts about the varieties already mentioned. Our main result is Theorem 3.6. In the following section we describe in greater detail the members of each equivalence class. There, all Urquhart's and Goldberg's results come into play, and we need to look carefully at the architecture of parts of the lattice of Ockham varieties.

The four equivalence classes above by no means exhaust $\Lambda(0)$. In Section 5 we exhibit a principal filter $\mathscr{F}$ in the lattice such that for every $\mathscr{A} \in \mathscr{F}$, every finite distributive lattice is the reduct of an algebra in $\mathscr{A}$. In addition, we include a discussion of the finite relative $\left(\mathbf{S} \vee \mathbf{S}^{d}\right.$ )-lattices, as an indication that the analysis of further $\approx$-equivalence classes raises interesting order-theoretic questions (involving the important classes of $\mathrm{N}$-free and series-parallel posets $([17],[21]))$. We shall pursue these questions in a subsequent paper.

Stone algebras lie at the confluence of two families of varieties-varieties of Ockham algebras and of distributive $p$-algebras. We conclude with some brief comments on finite relative $\mathscr{A}$-algebras when $\mathscr{A}$ is one of the subvarieties $\mathbf{B}_{n}$ of $\mathbf{B}_{\omega}$, the variety of all distributive $p$-algebras. (These varieties form a chain with $\mathbf{B}_{0}=\mathbf{B}$ and $\mathbf{B}_{1}=\mathbf{S}$.) For $n \geqslant 2$ it transpires that no worthwhile description in terms of forbidden subintervals is available. We obtain a similar negative result for $\mathbf{S} \vee \mathbf{S}^{d}$ : finite relative $\left(S \vee \mathbf{S}^{d}\right)$-lattices cannot be characterised by the exclusion of certain lattices as subintervals.

2. Preliminaries. The techniques used in [24] and [9] are purely algebraic. We shall use duality methods throughout. A topological duality for Ockham algebras was developed by A. Urquhart in [22], based on the duality for bounded distributive lattices. 
Since we are concerned in this paper only with finite algebras, topology plays no role, and we summarise the results we need in the special, purely order-theoretic, forms that pertain to the finite case. For a general survey of duality techniques, see B. A. Davey and D. Duffus [13] (which includes an introduction to Ockham algebras) or [19]. These articles give proofs of the unreferenced assertions below, or references to their proofs. We follow the terminology and notation of [19] when dealing with ordered sets. In particular, given an ordered (= partially ordered) set $P$, and a subset $Q$ of $P$, we let

$$
\downarrow Q=\{x \in P: \exists y \in Q \text { such that } x \leqslant y\}
$$

and call $Q$ decreasing (an alternative term is order ideal) if $\downarrow Q=Q$. We write $\downarrow x$ in place of $\downarrow\{x\}$. We define analogously $\uparrow Q, Q$ increasing and $\uparrow x$.

Underlying all that follows is the (full) duality between the category of finite distributive lattices (with $\{0,1\}$-preserving lattice homomorphisms) and the category of finite ordered sets (with order-preserving maps). At the object level this is just Birkhoff's classic representation theorem: any finite distributive lattice $L$ is isomorphic to the lattice of decreasing sets of $X_{L}$, where $X_{L}$ is the set $J(L)$ of join-irreducible elements of $L$ with the induced order. Given any ordered set $X$ we denote by $\mathscr{D}(X)$ the lattice of sets consisting of all decreasing subsets of $X$. Thus we have, for any finite distributive lattice $L$, that $L \cong \mathscr{D}\left(X_{L}\right)$. We shall henceforth identify $L$ with $\mathscr{D}\left(X_{L}\right)$.

Now let $L$ and $M$ be finite distributive lattices. Then there is a bijective correspondence $f \mapsto \phi_{f}$ between lattice homomorphisms (always assumed to preserve 0,1 ) $f: L \rightarrow M$ and order-preserving maps $\phi_{f}: X_{M} \rightarrow X_{L}$, with $f(a)=\phi^{-1}(a)$ for all $a \in M$. Further, $f$ is one-to-one if and only if $\phi_{f}$ is onto, and $f$ is onto if and only if $\phi_{f}$ is an order-embedding.

A finite Ockham algebra $L$ is a finite distributive lattice with an additional dual homomorphic operation $\sim$. We should therefore expect to obtain, by restricting the duality described above, a duality between the finite members of $\mathbf{O}$ and a suitable category of finite ordered sets-sets $X$ equipped with additional structure which defines on $\mathscr{D}(X)$. The appropriate dual category is that of finite Ockham spaces; we denote it by Q. We say $(X ; g)$ is an object in this category if $X$ is a finite ordered set and $g: X \rightarrow X$ is an order-reversing map (that is, $x \leqslant y$ implies $g(x) \geqslant g(y)$, for $x, y \in X)$. Then $\mathscr{D}(X)$ becomes an Ockham algebra once we define, for all $a \in \mathscr{D}(X)$,

$$
\sim a=X \backslash g^{-1}(a) \text {. }
$$

In the opposite direction, every finite Ockham algebra $L$ yields an order-reversing map defined on $X_{L}$ which satisfies the equation above. Morphisms in the dual category (called $g$-morphisms) are the order-preserving maps $\phi$ such that $\phi \circ g=g \circ \phi$. Proof that these definitions really do lead to a category equivalence can be found in [22].

Under this duality, the finite members of any subvariety $\mathscr{A}$ of $\mathbf{O}$ will correspond to a suitable (full) subcategory $\mathscr{y}^{\mathscr{A}}$ of $\mathscr{\mathscr { Y }}$. In every case the subcategory is specified by order-theoretic restrictions on the $g$-map. For Section 3 we need only the duals for the best known Ockham varieties, all of which are individually well known. Proposition 2.1 collects these together. See [1] for a systematic treatment of the process of translation between equational bases for Ockham varieties and $g$-inequalities on the spaces in the dual categories. 
Proposition 2.1. Let $L$ be a finite Ockham algebra with dual space $(X ; g) \in 9$. Then

(1) $L \in \mathbf{B}$ if and only if $g(x)=x$ for all $x \in X$;

(2) $L \in S$ if and only if $x \geqslant g(x)=g^{2}(x)$ for all $x \in X$;

(3) $L \in \mathbf{S}^{d}$ if and only if $x \leqslant g(x)=g^{2}(x)$ for all $x \in X$;

(4) $L \in M$ if and only if $x=g^{2}(x)$ for all $x \in X$;

(5) $L \in \mathbf{K}$ if and only if $x=g^{2}(x)$ and $x$ is comparable to $g(x)$ for all $x \in X$.

Now suppose $(X ; g) \in \mathscr{Y}$ is the space dual to the finite algebra $L \in \mathbf{O}$. We adopt the notation introduced by M. S. Goldberg $([15,16])$ and denote by $G(X)$ the collection of $g$-closed subsets of $X(Y$ is $g$-closed if $y \in Y$ implies $g(y) \in Y)$ and by $M(X)$ the collection of $g$-morphic images of $X$ (that is, images of $X$ by morphisms in $\mathscr{Y})$. The spaces in $\mathrm{G}(X)$ are the duals of the algebras in $\mathrm{H}(L)$, the homomorphic images (in $\mathbf{0}$ ) of $L$ and correspond to Ockham congruences on $L$, while $M(X)$ consists of spaces dual to elements of $\mathrm{S}(L)$, the Ockham subalgebras of $L$.

It follows from the observation made above concerning congruences that the algebra $L$ is subdirectly irreducible if and only if its dual space $(X ; g)$ is such that

$$
X=\left\{g^{k}(e): k=0,1,2, \ldots\right\} \text { for some } e \in X ;
$$

any such $e$ is known as an end of $X$. For details, see [22] or [16].

Each of the five varieties in Proposition 2.1 is of the form $A=\operatorname{HSP}(A)$, where $A$ is a finite subdirectly irreducible Ockham algebra. For such a variety $\mathscr{A}$, we denote by $S^{\mathscr{A}}=\left(X^{\mathscr{A}}, g^{\mathscr{A}}\right)$ the dual of the generating algebra $A$ of $\mathscr{A}$. For the aforementioned varieties we can depict these spaces as follows:

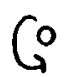

$S^{\mathbf{B}}$
$S^{\mathbf{S}}$

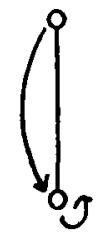

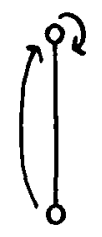
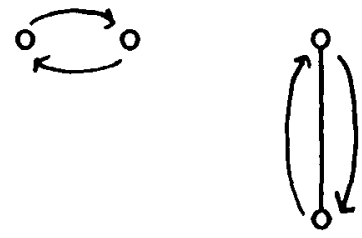

$S^{\mathbf{M}}$

$S^{\mathbf{K}}$

Figure 1

3. Relative Ockham lattices. Let us begin by establishing some notation connected with the concepts introduced in Section 1.

DefinITION 3.1. Let $A$ be a variety of Ockham algebras and let $L$ be a finite distributive lattice. Then $L$ is a relative $\mathscr{A}$-lattice if and only if every interval $[a, b]$ of $L$ can be given the structure of an algebra in $\mathscr{A}$. The class of finite relative $\mathscr{A}$-lattices is denoted by $\mathscr{L}^{\mathscr{A}}$. The equivalence relation $\approx$ is defined on $\Lambda(0)$ by $\mathscr{A} \approx \mathscr{B}$ if and only if $\mathscr{L}^{\star}=\mathscr{L}^{\mathscr{A}}$. The equivalence class of $\mathscr{A}$ is denoted by [ $\mathbb{A}$ ].

Lemma 3.2, which appears not to have been recorded in the existing literature, provides the key to handling subintervals in finite distributive lattices. Before stating this 
lemma, we draw attention to two different ways in which one ordered set may lie inside another. Let $P$ and $Q$ be (finite) ordered sets. We say that

(i) $P$ has $Q$ as a subposet if there exists an order-embedding $\eta$ of $Q$ into $P$,

(ii) $P$ has $Q$ as a convex subposet if there exists an order-embedding $\eta: Q \rightarrow P$ such that $\eta(Q)$ is a convex subset of $P$.

(A subset $S$ of an ordered set $P$ is convex if $u, v \in S, x \in P$ and $u \leqslant x \leqslant v$ imply $x \in S$.)

We shall adopt the notational convention due to I. Rival [21] and use alphabetic symbols for certain ordered sets. Accordingly, the letters $\mathbf{N}$ and $\mathbf{V}$ are used to denote respectively

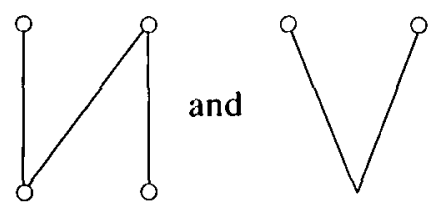

In the same way we shall use $\mathbf{I}$ and $\boldsymbol{\Lambda}$ for, respectively,

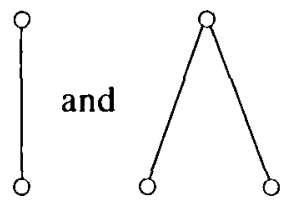

To illustrate our definitions, we observe that the ordered set in Fig. 2 has $\mathbf{N}$ as a subposet but not as a convex subposet.

Our characterisations of classes of relative Ockham lattices will initially be in terms of the exclusion of certain ordered sets from the dual spaces of the lattices concerned, either as subposets or as convex subposets.

LeMMA 3.2. Let $L$ be a finite distributive lattice.

(1) Let $M=[a, b]$ be a closed subinterval of $L$. Then $X_{L}$ has $X_{M}$ as a convex subposet.

(2) Let $Q$ be a convex subset of $P$. Then there exists a closed subinterval $M$ of $L$ such that $X_{M}$ is order-isomorphic to $Q$.

Proof. Take decreasing subsets $a$ and $b$ of $X_{L}$ with $a \subseteq b$. We first claim that $b \backslash a$ is a convex subset of $P$. Suppose $u, v \in b \backslash a$ and $u \leqslant x \leqslant v$. Since $b$ is decreasing and contains $v$, the point $x$ must lie in $b$. On the other hand, because the complement of $a$ is increasing and contains $u$, we must have $x \notin a$. This establishes the convexity of $b \backslash a$.

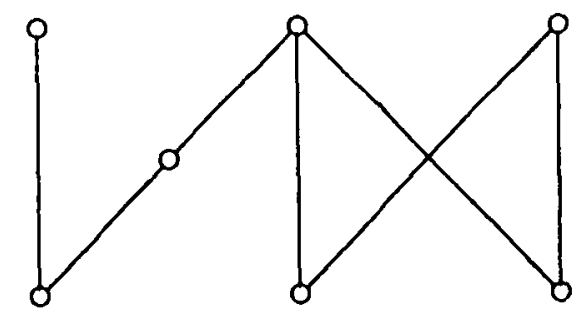

Figure 2 
We next show that $\mathscr{D}(b \backslash a)$ is isomorphic to $M=[a, b]$. For each $c \in \mathscr{D}(b \backslash a)$, define $f(c)=c \cup a$. Then it is easily seen that $f(c)$ is decreasing and that $a \subseteq f(c) \subseteq b$, so that $f(c) \in M$. Further $f$ is a one-to-one map with image $M$, and is trivially a lattice homomorphism. This completes the proof of (1).

Conversely, take a convex subset $Q$ of $P$. Convexity of $Q$ is equivalent to the condition $Q=\downarrow Q \cap \uparrow Q$. Hence

$$
\begin{aligned}
Q & =\downarrow Q \backslash(\downarrow Q \backslash Q) \\
& =\downarrow Q \backslash(\downarrow Q \backslash(\downarrow Q \cap \uparrow Q)) \\
& =\downarrow Q \backslash\left(\downarrow Q \cap\left(X_{L} \backslash \uparrow Q\right)\right) .
\end{aligned}
$$

The sets $b=\downarrow Q$ and $a=\downarrow Q \cap\left(X_{L} \backslash \uparrow Q\right)$ are both decreasing. Thus $Q$ is order-isomorphic to $X_{M}$, where $M=[a, b]$.

We note that the correspondence Lemma 3.2 establishes between intervals of $L$ and convex subposets of $X_{L}$ is not one-to-one. Many different intervals may give rise to the same convex set.

We shall say that a class $\mathscr{E}$ of finite ordered sets is convex-closed if whenever $P$ belongs to $\mathscr{E}$, every convex subposet of $P$ also belongs to $\mathscr{E}$.

It is now possible to outline our fundamental strategy. A finite distributive lattice $L$ is a relative $\mathscr{A}$-lattice $(\mathscr{A} \in \Lambda(\mathbf{0}))$ if and only if every convex subposet of $X_{L}$ can be endowed with a $g$-map making it into a space in $\mathscr{y}^{\mathscr{A}}$. We seek a family $\left\{E_{i}\right\}_{i \in I}$ of ordered sets, in some sense minimal, such that none of the sets $E_{i}$ can be made into a space in $g^{*}$. We may then consider the class $\mathscr{E}$ of ordered sets which fail to have any of the sets $E_{i}$ as a convex poset. Suppose that every member of $\mathscr{E}$ can be made into a member of $\mathscr{G} \mathscr{}$ and that $\mathscr{E}$ is convex-closed. Then we can conclude that the finite relative $\mathscr{A}$-lattices are exactly those $L$ for which $X_{L} \in \mathscr{E}$. Actually we can assert a little more. The lattices $L$ with $X_{L} \in \mathscr{E}$ will be the finite relative $\mathscr{B}$-algebras for any variety $\mathscr{B}$ containing $\mathscr{A}$ which is such that no set $E_{i}$ can be made into the dual of an algebra in $\mathscr{B}$. It is this last observation which will enable us to describe certain equivalence classes under the equivalence relation $\approx$.

To handle the varieties $\mathbf{B}, \mathbf{S}, \mathbf{S}^{d}$ and $\mathbf{K}$ we need to consider $\mathbf{I}, \boldsymbol{\Lambda}$ and $\mathbf{V}$ as excluded posets. Our first observation is a triviality, which we state as a lemma to bring it into line with the slightly less trivial lemmas which follow it.

Lemma 3.3. Let $P$ be a finite ordered set. Then the following are equivalent:

(1) $P$ is an antichain;

(2) $P$ fails to contain I as a subposet;

(3) $P$ fails to contain I as a convex subposet.

The class of all finite antichains is convex-closed.

LEMMA 3.4. Let $P$ be a finite ordered set. Then the following are equivalent:

(1) $P$ is a disjoint union of trees (that is, $\downarrow x$ is a chain for each $x \in P$ );

(2) $P$ fails to contain $\Lambda$ as a subposet;

(3) $P$ fails to contain $\Lambda$ as a convex subposet.

The class of all disjoint unions of finite trees is convex-closed.

Proof. Trivially (1) is equivalent to (2) and (2) implies (3). We prove that (3) implies (2) by showing that if $P$ contains points $x, u, v$ with $x>u, x>v$ and $u \| v(u, v$ are 
incomparable) then $P$ also has a convex subposet order-isomorphic to $\Lambda$.

Let $x^{\prime}$ be a minimal element of the (non-empty) set $\downarrow x \cap(\uparrow u \cap \uparrow v)$. Then $x^{\prime}>u$, $x^{\prime}>v$ and there does not exist $y$ such that $x^{\prime}>y, y>u$ and $y>v$. Now choose $u^{\prime}$ to be a maximal element of $\left(\downarrow x^{\prime} \backslash\left\{x^{\prime}\right\}\right) \cap \uparrow u$ and (similarly) $v^{\prime}$ to be a maximal element of $\left(\downarrow x^{\prime} \backslash\left\{x^{\prime}\right\}\right) \cap \uparrow v$. We claim $Q=\left\{x^{\prime}, u^{\prime}, v^{\prime}\right\}$ is convex as a subset of $P$ and is isomorphic to $\boldsymbol{\Lambda}$. The convexity is a consequence of the maximality conditon on $u^{\prime}, v^{\prime}$. We have $x^{\prime}>u^{\prime} \geqslant u$ and $x^{\prime}>v^{\prime} \geqslant v$, by construction. We require $u^{\prime} \| v^{\prime}$. Suppose otherwise. Then without loss of generality $u^{\prime} \geqslant v^{\prime}$. In this case $u^{\prime} \in \uparrow u \cap \uparrow v \cap \downarrow x^{\prime}$, which is impossible by the minimality condition on $x^{\prime}$.

Lemma 3.4 has an obvious (order-theoretic) dual, concerning the exclusion of $\mathbf{V}$. If we combine this dual version with Lemma 3.4 we get the next lemma.

Lemma 3.5. Let $P$ be a fimite ordered set. Then the following are equivalent:

(1) $P$ is a disjoint union of chains;

(2) $P$ fails to contain either $\Lambda$ or $\mathbf{V}$ as a subposet;

(3) $P$ fails to contain either $\Lambda$ or $\boldsymbol{V}$ as a convex subposet.

We can now prove the main theorem of this section.

THEOREM 3.6.

(1) $[\mathbf{B}]=\left\{\mathscr{A} \in \Lambda(\mathbf{O}): \mathscr{A} \supseteq \mathbf{B}, \mathscr{A} \nsupseteq \mathbf{K}, \mathscr{A} \nsupseteq \mathbf{S}\right.$ and $\left.\mathscr{A} \nsupseteq \mathbf{S}^{d}\right\}$;

(2) $[\mathbf{K}]=\left\{\mathscr{A} \in \Lambda(\mathbf{O}): \mathscr{A} \supseteq \mathbf{K}, \mathscr{A} \notin \mathbf{S}\right.$ and $\left.\mathscr{A} \notin \mathbf{S}^{d}\right\}$;

(3) $[\mathbf{S}]=\left\{\mathscr{A} \in \Lambda(\mathbf{O}): \mathscr{A} \supseteq \mathbf{S}\right.$ and $\left.\mathscr{A} \notin \mathbf{S}^{d}\right\}$;

(4) $\left[\mathbf{S}^{d}\right]=\left\{\mathscr{A} \in \Lambda(\mathbf{O}): \mathscr{A} \supseteq \mathbf{S}^{d}\right.$ and $\left.\mathscr{A} \nsupseteq \mathbf{S}\right\}$.

Proof. (1). Let $P$ be a finite ordered set. Then $P$ fails to be an antichain if and only if $P$ contains $I$ as a convex subposet. There are three possible $g$-maps on $I$. These make it into $X^{\mathbf{K}}, X^{\mathbf{S}}$ or $X^{\mathbf{S}^{d}}$. But for any $\mathscr{B}, X^{\mathscr{B}}$ belongs to $\mathscr{Y}^{\mathscr{A}}$ if and only if $\mathscr{B} \subseteq \mathscr{A}$. (This is simply the dual of the assertion that if $\mathscr{A}$ is any variety, an algebra $A$ belongs to $\mathscr{A}$ if and only if $\operatorname{HSP}(A) \subseteq \mathscr{A}$.)

Suppose $P$ is an antichain. Then $P$ becomes an element of $X^{\mathbf{B}}$ if it is endowed with the identity map as $g$-map. Hence $P$ is the dual of an algebra in $\mathscr{A}$ for any variety $\mathscr{A}$.

(2). We need to consider the possible $g$-maps on $\wedge$. Consider an ordered set $Q=\{x, u, v\}$ with $x>u, x>v$ and $u \| v$. Suppose $g: Q \rightarrow Q$ is order-reversing. If $g(x)=x$, then necessarily $g(u)=g(v)=x$. If $g(x)=u$, then $g(u)=u$ or $g(u)=x$. In each of these cases we may have either $g(v)=u$ or $g(v)=x$. Consequently we have five different $g$-maps on $\wedge$, as shown in Fig. 3 .

Each of these Ockham spaces has $X^{\mathbf{S}^{d}}$ as a $g$-morphic image.
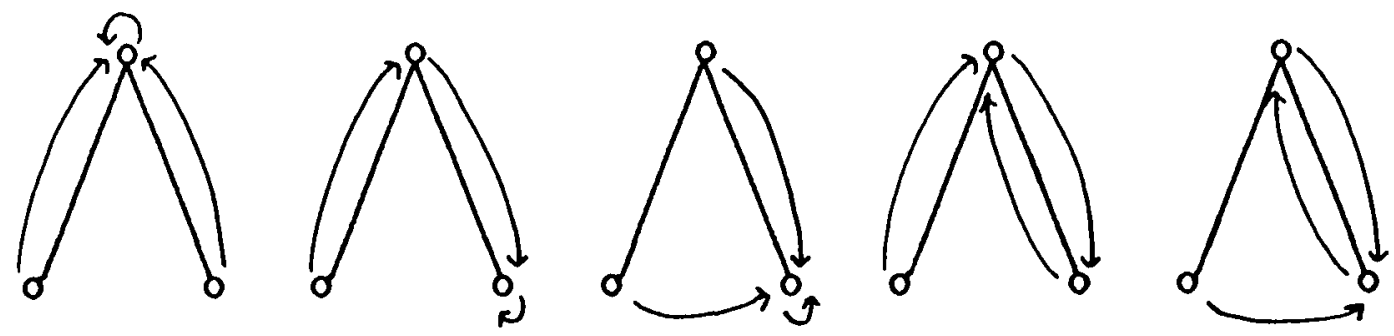

Figure 3 
Suppose $P$ has $\Lambda$ as a convex subposet and is the dual of a relative $\mathscr{A}$-lattice. Then (by Lemma 3.5) it must be possible to make $\Lambda$ into the dual of an algebra $M$ in $\mathscr{A}$. When this is done, $A$ contains every Ockham subalgebra of $M$, and one of these subalgebras must be the generating algebra for $S^{d}$. It follows that $\mathscr{A} \supseteq S^{d}$. Thus if $\mathscr{A} \notin \mathbf{S}^{d}$, the dual of any finite relative $\mathscr{A}$-lattice fails to contain $\Lambda$. In the same way, if $\mathscr{A} \notin \mathbf{S}$, the dual of any finite relative $\mathscr{A}$-lattice fails to contain $\mathbf{V}$. We conclude that if $\mathscr{A}$ contains neither $\mathbf{S}$ nor $S^{d}$ then every finite relative $\mathscr{A}$-lattice $L$ is such that $X_{L}$ is a disjoint union of chains.

Let $P$ be the chain $1<2<\cdots<k$. Define $g$ by $g(i)=k-i+1$. Then $g$ is order-reversing and such that $g^{2}$ is the identity. Trivially each point is comparable to its $g$-image. Now suppose $P$ is a disjoint union of finite chains. Then a $g$-map can be defined on $P$ by using the construction above on each order component. Proposition 2.1 now implies that any disjoint union of finite chains can be made into the dual space of a Kleene algebra. This completes the proof of (2).

(3) Note that any disjoint union of finite trees can be made into the dual of an algebra in $\mathscr{A}$ whenever $\mathscr{A}$ is an Ockham variety containing $\mathbf{S}$. The appropriate $g$-map simply takes every element to the (unique) minimal point of its order component. The remainder of the argument required to prove (3) is as in the proof of (2).

(4) This final part is merely the order dual of (3).

Our final goal in this section is to give an algebraic description of $\mathscr{L}^{\mathscr{A}}$ for $\mathscr{A}$ in any of the equivalence classes in Theorem 3.6. The constructive characterisation of finite relative Stone algebras provided by Theorem 3.7(iii) appears not to have been stated before.

THeOREM 3.7. Let $\mathscr{A} \in \Lambda(\mathbf{0})$ and $L$ be a finite distributive lattice.

(i) If $\mathscr{A} \in[\mathbf{B}]$, then $L \in \mathscr{L}^{\mathscr{A}}$ if and only if $L$ is Boolean;

(ii) If $\mathscr{A} \in[\mathbf{K}]$, then $L \in \mathscr{L}^{\mathfrak{A}}$ if and only if it satisfies any of the following equivalent conditions:

(1) $L$ is a direct product of chains;

(2) L fails to contain either $2^{2} \oplus 1$ or $1 \oplus 2^{2}$ as a subinterval;

(3) L fails to have either $2^{2} \oplus 1$ or $1 \oplus 2^{2}$ as a homomorphic image;

(iii) If $\mathscr{A} \in[\mathrm{S}]$, then $\mathscr{L}^{\mathscr{S}}$ is defined in the following way:

(a) the trivial algebra and the 2-element chain are in $\mathscr{L}^{\text {s }}$,

(b) any finite direct product of elements of $\mathscr{L}^{\star s}$ is in $\mathscr{L}^{\star}$,

(c) if $L \in \mathscr{L}^{\mathscr{A}}$, then $1 \oplus L \in \mathscr{L}^{\star}$,

(d) any element of $\mathscr{L}^{\mathscr{A}}$ can be constructed by repeated application of $(a),(b)$ and (c).

Further, $L \in \mathscr{L}^{\text {st }}$ if and only if it satisfies either of the following equivalent conditions:

(1) $L$ fails to contain $2^{2} \oplus 1$ as a subinterval;

(2) L fails to have $2^{2} \oplus 1$ as a homomorphic image.

(iv) If $\mathscr{A} \in\left[\mathbf{S}^{d}\right]$, then $\mathscr{L}^{\mathscr{A}}$ is defined in the following way:

(a) the trivial algebra and the 2-element chain are in $\mathscr{L}^{\mathscr{A}}$,

(b) any finite direct product of elements of $\mathscr{L}^{x}$ is in $\mathscr{L}^{\star}$,

(c) if $L \in \mathscr{L}^{*}$, then $L \oplus \mathbf{1} \in \mathscr{L}^{*}$,

(d) any element of $\mathscr{L}^{*}$ can be constructed by repeated application of (a)-(c).

Further, $L \in \mathscr{L}^{\mathcal{A}}$ if and only if it satisfies either of the following equivalent conditions:

(1) L fails to contain $1 \oplus \mathbf{2}^{2}$ as a subinterval;

(2) L fails to have $1 \oplus \mathbf{2}^{2}$ as a homomorphic image. 
Proof. Almost all the assertions in the theorem are dual versions of earlier results. Only the first part of (iii) and the first part of (iv) need proof, and it is enough to prove the former. For this it is sufficient to show that the class $\mathscr{T}$ of finite ordered sets generated by the duals of (a)-(d) in (iii) is exactly the class of disjoint unions of finite trees. We have that $\mathscr{T}$ is the smallest class of ordered sets containing the empty set and any singleton, and closed under disjoint unions and the operation of adjoining a universal minimal point. An elementary inductive argument shows that the ordered sets so defined are indeed just the disjoint unions of finite trees.

The starting point for our investigations was Varlet's characterisation of finite relative de Morgan lattices. Since the variety $\mathbf{M}$ contains $\mathbf{K}$ but neither $\mathbf{S}$ nor $\mathbf{S}^{d}$, it belongs to [K]. Theorem 3.6(2) and Theorem 3.7(2) encompass Varlet's result, and show that $\mathbf{M}$ plays no special role in the general scheme of relative Ockham lattices. The next section addresses the question of exactly which Ockham varieties comprise the various equivalence classes so far considered.

We remark that each of the equivalence classes we have described has a smallest element, which we have taken as the representative of the class. Let $\mathscr{C}$ denote any of these four varieties. Then every lattice in $\mathscr{L}^{\mathscr{C}}$ can be made into an algebra in $\mathscr{C}$ in just one way. This is not true in general. The lattice $3 \times 3$ belongs to $\mathscr{L}^{\mathrm{M}}$ and has two de Morgan structures, one making it into an element of $\mathbf{K}$ and another making it an element of $\mathbf{M} \backslash \mathbf{K}$. The second is specified by the $g$-map on the dual $2 \cup 2$ shown in Fig. 4 .

4. The lattice of Ockham subvarieties. In Section 3 we needed very little knowledge of the lattice $\Lambda(\mathbf{0})$. We now seek to gain a greater insight into those results by investigating the structure of $\Lambda(0)$ more closely.

Underpinning what follows is one key fact: $\mathbf{O}$ is congruence-distributive. This can be proved in a number of ways. It is, in particular, an immediate consequence of the fact that the join operation in the congruence lattice of any algebra $A \in \mathbf{O}$ is the same as the join operation in the (distributive) congruence lattice of the distributive lattice reduct of $A$; see [22]. Congruence-distributivity allows the application of Jónsson's lemma and its many consequences (see $[\mathbf{1 8}]$ ).

Any element of $\Lambda_{f}(\mathbf{O})$ may be presented in any one of the following equivalent ways:

(1) $\mathscr{A}=\operatorname{HSP}\left(A_{1}, \ldots, A_{k}\right)$, where $A_{1}, \ldots, A_{k}$ are finite subdirectly irreducible Ockham algebras.

(2) $\mathscr{A}=\mathrm{HSP}\left(A_{1}\right) \vee \ldots \vee \operatorname{HSP}\left(A_{k}\right)$, where $A_{1}, \ldots, A_{k}$ are finite subdirectly irreducible algebras.

(3) $\mathscr{A}=\mathrm{HSP}(A)$, where $A$ is finite, but not necessarily subdirectly irreducible.

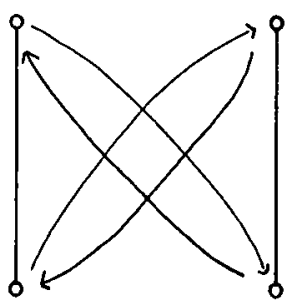

Figure 4 
For the third one, just consider $\left(A_{1} \times \ldots \times A_{k}\right)$. In any congruence-distributive variety $\mathscr{A}$ a subvariety generated by a finite algebra is of finite height in $\Lambda(\mathscr{A})$. We note in passing that in [23] A. Urquhart shows the converse also holds in $\mathbf{0}$.

The next theorem contains many of the essential tools for working with $\Lambda_{f}(\mathbf{O})$. It is an amalgam of results from M. S. Goldberg [16, Section 2] and B. A. Davey [12].

Theorem 4.1. Suppose $\mathscr{A}=\operatorname{HSP}\left(A_{1}, A_{2}, \ldots, A_{k}\right)$ (where $A_{1}, \ldots, A_{k}$ are subdirectly irreducible) belongs to $\Lambda_{f}(\mathbf{O})$. Then the set $\mathrm{Si}(\mathscr{A})$ of subdirectly irreducible algebras in $\mathscr{A}$ coincides with $\mathrm{HS}\left(A_{1}, \ldots, A_{k}\right)$.

Let $\mathrm{Si}(\mathscr{A})$ be ordered by $A \leqslant B$ if and only if $A \in \mathrm{HS}(B)$. Then the lattice $\Lambda(\mathscr{A})$ of subvarieties of $A$ is isomorphic to $\mathscr{D}(\operatorname{Si}(\mathscr{A}))$.

As observed in Section 2, the finite Ockham algebra $A$ is subdirectly irreducible if and only if its dual space has an end. Let $\left\{e, g(e), \ldots, g^{m-1}(e)\right\}$, with $g^{n}(e)=g^{m}(e)$ $(m>n \geqslant 0)$, be such a dual space. This space may carry any order relation with respect to which $g$ is order-reversing. When the order is discrete, the corresponding algebra is denoted $L_{m, n}$ and the variety it generates by $\mathbf{P}_{m, n}$. These varieties are important in that they form a 'skeleton' for $\Lambda_{f}(\mathbf{O})$ : every element of $\Lambda_{f}(\mathbf{O})$ lies in some $\mathbf{P}_{m, n}(m>n \geqslant 0)$. Equationally the variety $\mathbf{P}_{m, n}$ is defined within $\mathbf{O}$ by

$$
\begin{aligned}
& \sim^{m} a=\sim^{n} a \quad \text { ( } m-n \text { even) } \\
& \sim^{m} a \wedge \sim^{n} a=0 \text { and } \quad \sim^{m} a \vee \sim^{n} a=1 \quad(m-n \text { odd })
\end{aligned}
$$

(see A. Urquhart [22] and M. S. Goldberg [16]). In the case $m-n$ even, the varieties $\mathbf{P}_{m, n}$ were introduced and studied earlier by J. Berman [3], using the notation $\mathbf{K}_{p, q}$ for $\mathbf{P}_{2 p+q, q}$.

The variety of MS-algebras is the subvariety of $\mathbf{P}_{3,1}$ consisting of algebras satisfying $a \leqslant \sim^{2} a$. Its lattice of subvarieties was obtained by T. S. Blyth and J. C. Varlet in [7], using Theorem 4.1. From the diagram of $\Lambda(\mathbf{M S})$ in [7] we see immediately that all the non-trivial MS-subvarieties lie in [S] except $\mathbf{B}$ and the four varieties $\mathbf{K}, \mathbf{M}, \mathbf{K}_{1}$ and $\mathbf{M} \vee \mathbf{K}_{1}$, each of which lies in [K]. Here $\mathbf{K}_{1}$ is the variety generated by the 4-element chain $0<a<b<1$ with $\sim a=\sim b=b$; the dual space of this chain is the 3-element chain $g^{2}(e)<e<g(e)=g^{3}(e)$.

Applying Theorem 3.6 directly in this way is only practical for very small subvarieties of $\mathbf{0}$. The size of $\Lambda\left(\mathbf{P}_{m, n}\right)$ grows extremely rapidly with $m$ and $n$. Already $\Lambda\left(\mathbf{P}_{3,1}\right)$ has 403 elements (see [5]).

We therefore seek to analyse the equivalence classes under $\approx$ determined by Theorem 3.6 without recourse to drawing sublattices of $\Lambda_{f}(\mathbf{0})$. Let $A_{1}, \ldots, A_{k}$ and $B$ be finite subdirectly irreducible Ockham algebras. Then, by the first part of Theorem 4.1 and duality, we have $\operatorname{HSP}\left(A_{1}, \ldots, A_{k}\right) \supseteq \operatorname{HSP}(B)$ if and only if $X_{B} \in \operatorname{GM}\left(X_{A_{i}}\right)$ for some $i=1, \ldots, k$. (Here $\mathrm{GM}(X)$ denotes the set of $g$-closed subsets of $g$-morphic images of the Ockham space $X$; because $\mathbf{0}$ has the Congruence Extension Property, $\operatorname{GM}(X)=$ $\operatorname{MG}(X)$.)

Take $X=\left\{e, g(e), \ldots, g^{m-1}(e)\right\}$, with $g^{n}(e)=g^{m}(e)$, to be the dual of some finite subdirectly irreducible algebra. The set $\left\{g^{n}(e), \ldots, g^{m-1}(e)\right\}$ is called the loop of $X$, and $\left\{e, g(e), \ldots, g^{n-1}(e)\right\}$ the tail. From the following observations about $\operatorname{GM}(X)$ we can determine under what circumstances each of $X^{\mathbf{K}}, X^{\mathbf{s}}$ and $X^{\mathbf{S}^{d}}$ belongs to $\operatorname{GM}(X)$. 
(1) $\mathrm{G}(X)$ consists of the empty set and the sets $\left\{g^{r}(e), \ldots, g^{m-1}(e)\right\}$, for $0 \leqslant r \leqslant n$.

(2) Any $g$-morphism with domain $X$ maps the loop of $X$ onto the loop of the image space, and the size of the image loop divides $m-n$.

(3) If $m-n$ is odd, then the loop of $X$ is discretely ordered. If $m-n$ is even, then the loop is of height at most one [A. Urquhart [22], or see M. S. Goldberg [16]].

(4) Call a point $g^{r}(e)$ of $X$ trapped if there exist distinct $k, l$ with $n \leqslant k, l \leqslant m-1$ such that $g^{k}(e) \leqslant g^{r}(e) \leqslant g^{l}(e)$. The set of trapped points is a $g$-closed subset of $X$ containing the loop. Contracting all trapped points to a single point yields a $g$-morphic image of $X$.

The significance of trapping is that if the loop is contracted to a point, as it must be if $X^{\mathbf{S}}$ or $X^{\mathbf{S}^{d}}$ is to be obtained as an element of $\operatorname{GM}(X)$, then all trapped points perforce map to the same point. The simplest example of non-trivial trapping of an end is provided by the dual space of the generating algebra of the MS-variety $\mathbf{K}_{1}$ mentioned above.

LemmA 4.2. Let $X$ be as above, $|X| \geqslant 2$.

(i) $X^{\mathbf{K}}$ belongs to $\mathrm{GM}(X)$ if and only if $m-n$ is even.

(ii) Neither $X^{\mathbf{S}}$ nor $X^{\mathbf{S}^{d}}$ belongs to $\mathrm{GM}(X)$ if and only if the end of $X$ is trapped.

(iii) $X^{\mathbf{S}}$ belongs to $\mathrm{GM}(X)$ but $X^{\mathbf{S}^{d}}$ does not if and only if $n=1$ and, with respect to the order on $X$, the end is a maximal point which is not also a minimal point (and dually).

Proof. Parts (i) and (ii) are direct consequences of the observations (1)-(4) above.

For (iii), note that if neither $e$ nor $g(e)$ is trapped, then $\left\{g^{2}(e), \ldots, g^{m-1}(e)\right\}$ can be contracted to a point. Then the resulting space $Y$ is such that both $X^{\mathbf{s}}$ and $X^{\mathbf{s}^{d}}$ lie in $\mathrm{GM}(Y)$ and so in $\mathrm{GM}(X)$. Now suppose that $e$ is the only point which is not trapped. Contracting $X \backslash\{x\}$ to a point yields one of $X^{\mathbf{s}}, X^{\mathbf{S}^{d}}$, or $X^{\mathbf{P}_{2,1}}$. We deduce that we have both $X^{\mathbf{s}} \in \mathrm{GM}(X)$ and $X^{\mathbf{s}^{d}} \notin \mathrm{GM}(X)$ only if $e$ lies above some point in $\left\{g(e), \ldots, g^{m-1}(e)\right\}$ and is maximal. Because $g$ is order-reversing, $g(e)$ must then be minimal. By hypothesis it is trapped, so $g(e)$ actually belongs to the loop. Thus $n=1$. The converse is obvious.

The rather mysterious conditions in (ii) and (iii) above translate into algebraic conditions involving congruences. The congruence lattice of a finite Ockham algebra is anti-isomorphic to the lattice of $g$-closed subsets of its dual space, via $\theta \mapsto G_{\theta}$, where $a \equiv b(\theta)$ if and only if $a \cap G_{\theta}=b \cap G_{\theta}$; see [22]. The congruence class of $a$ under $\theta$ is denoted by $[a]_{\theta}$.

LEMMA 4.3. Let $A$ be as above and let $\theta$ be any non-trivial congruence on $A$. Then

(i) $\operatorname{HSP}(A)$ contains neither $\mathbf{S}$ nor $\mathbf{S}^{d}$ if and only if $\left|[0]_{\theta}\right|=\left|[1]_{\theta}\right|=1$;

(ii) $\operatorname{HSP}(A)$ contains $\mathbf{S}$ but not $\mathbf{S}^{d}$ if and only if $\left|[0]_{\theta}\right|=2$ and $\left|[1]_{\theta}\right|=1$.

Proof. Let $\theta$ be any non-trivial congruence. For (i) note that $\left|[0]_{\theta}\right|=1$ is a way of expressing the condition that every non-empty increasing subset of the dual of $A$ intersects every non-empty $g$-closed subset. The second part uses the fact that $[1]_{\theta}$ contains two elements precisely when the $g$-closed subset corresponding to $\theta$ misses exactly one maximal point.

Proposition 4.4. Let $\mathscr{A} \in \Lambda_{f}(\mathbf{O})$. Then $\mathscr{A} \in[\mathrm{B}]$ if and only if $\mathscr{A}$ is a finite join of varieties of the form $P_{m, 0}$ with $m$ odd. 
Proof. Write $\mathscr{A}=\mathrm{HSP}\left(A_{1}\right) \vee \ldots \vee \mathrm{HSP}\left(A_{k}\right)$ and suppose $\mathscr{A} \in[\mathrm{B}]$. Theorem 3.6 and Lemma 4.2(i) imply that the dual of each $A_{i}$ must have a loop which is of odd length and discretely ordered. By Lemma 4.2(ii) we also require that each of these spaces has a trapped end. This happens if and only if each space is itself a loop. Hence has to be of the stated form. The converse is obvious.

The varieties arising in Proposition 4.4 have a number of alternative descriptions. Note that if $m$ is odd then the join-irreducible elements of $\Lambda\left(\mathbf{P}_{m, 0}\right)$ are exactly the varieties $\mathbf{P}_{k, 0}$ where $k$ divides $m$. This implies that the members of $[\mathbf{B}] \cap \Lambda_{f}(\mathbf{0})$ are precisely those varieties which lie in some $\mathbf{P}_{m, 0}(m$ odd) or, equivalently, are the varieties $\mathbf{P}_{m, 0}(m$ odd) and their lower covers in $\Lambda(\mathbf{O})$. (These equivalences are essentially due to A. Urquhart [22] and M. S. Goldberg [15].)

The situation for other equivalence classes is more complicated because there is a multitude of ways in which points of the dual of a finite subdirectly irreducible algebra can get trapped. We do not attempt to present a complete list of the elements of $[\mathscr{A}] \cap \Lambda_{f}(\mathbf{0})$ for $\mathscr{A}=\mathbf{K}, \mathbf{S}$ or $\mathbf{S}^{d}$. However it is easy to use Lemma 4.2 to exhibit varieties which lie in the various classes.

Suppose next that $X$ satisfies the conditions of Lemma 4.2(iii) and assume in addition that the loop of $X$ is an antichain. For some $r$ with $m>r \geqslant 1, e>g^{r}(e)$. This implies $g(e) \leqslant g^{r+1}(e)$, whence $g(e)=g^{r+1}(e)$ since both points belong to the loop. Hence we must have $r=m-1$, and this choice is certainly allowable. Let $\mathscr{A}^{(m)}$ be the variety in [S] generated by the subdirectly irreducible algebra whose dual is $X$.

Now suppose $m$ is odd, $m=2 p+1$. The techniques collected together in [1] reveal that $\mathscr{A}^{(2 p+1)}$ is the subvariety $\mathbf{M S}^{(p)}$ of $\mathbf{P}_{2 p+1,1}$ given by $a \leqslant \sim^{2 p} a$. We have here just the family of varieties of generalised MS-algebras introduced by $M$. Ramalho and $M$. Sequeira in [20]; $p=1$ gives the MS-algebras. (The restriction that $m$ be odd is not essential. However the variety arising from $X$ has a different form of equational base in case $m$ is even.)

Proposition 4.5. [S] $\cap \Lambda_{f}(\mathbf{O})$ contains $\mathbf{M S}^{(p)}$ for every $p . A$ variety $\mathscr{A}$ lies in $[\mathbf{S}] \cap \Lambda_{f}(\mathbf{O})$ if and only if it is a finite join of varieties each of which is a non-trivial subvariety of some $\mathscr{A}^{(m)}(m \geqslant 1)$ generated by a single subdirectly irreducible algebra the end of whose dual space is not trapped.

Proof. Almost everything follows from Theorem 3.6 and Lemma 4.2. The proof is completed by showing that any space $X$ meeting the conditions of Lemma 4.2(iii) must be a $g$-morphic image of a space $\left\{e, g(e), \ldots, g^{m-1}(e)\right\}$ in which the only comparability is $e>g^{m-1}(e)$. When $m$ is even $X$ is itself such a space, so assume $m$ is odd. There exists $r$ with $1 \leqslant r \leqslant m-1$ such that $e>g^{r}(e)$. Since $e$ is maximal, $g^{k}(e)$ is minimal if $k$ is odd and maximal if $k$ is even. Hence $r$ is odd, so $g^{m-r}$ is order-preserving. Therefore $g^{m-r}(e) \leqslant g^{m}(e)=g(e)$. Because $g(e)$ is minimal, $g^{m-r}(e)=g(e)$. This forces $r=m-1$, whence $X$ must be of the required form.

Finally we look at varieties in the equivalence class of $\mathbf{K}$.

Proposition 4.6. $[\mathbf{K}] \cap \Lambda_{f}(\mathbf{O})$ contains all varieties $\mathbf{P}_{m, 0}$ ( $m$ even). More generally, it contains any variety which is a finite join of non-trivial varieties each of which is a subvariety (other than $\mathbf{B}$ ) of some $\mathbf{P}_{m, 0}$ (m even). In addition, for each $s \geqslant 3,[\mathbf{K}] \cap \Lambda_{f}(\mathbf{O})$ 
contains a variety generated by a subdirectly irreducible algebra which is a chain with $s$ elements.

Proof. The first part is derived from Theorem 3.6 and Lemma 4.2. For the second part we just need to construct an Ockham space with $s-1$ elements which is a chain, which has a trapped end, and which has a 2-element loop. Suppose $s=2 q$ and define a $g$-map on the chain $\mathbf{k}$, where $k=2 q-2$, by

$$
g(i)= \begin{cases}2 q-i-1 & \text { if } k \in\{0, \ldots, q-1\} \cup\{2 q-1\} \\ 2 q-i-2 & \text { if } k \in\{q, \ldots, 2 q-2\}\end{cases}
$$

(here $q-1$ is the end). The case that $s$ is odd is handled similarly.

A. Urquhart proves in [23] that $\Lambda(\mathbf{O})$ is uncountable. On the other hand, $\Lambda_{f}(\mathbf{O})$ is countably infinite, since each finite Ockham algebra lies in some $\mathbf{P}_{m, n}$. It follows from the results above that each of the equivalence classes $[\mathbf{B}],[\mathbf{K}],[\mathbf{S}]$ and $\left[\mathbf{S}^{d}\right]$ intersects $\Lambda_{f}(\mathbf{O})$ in a countably infinite set. Further, each class contains elements of $\Lambda(\mathbf{O})$ of arbitrarily large finite height. Each also contains elements which are joins of join-irreducibles forming arbitrarily large finite antichains in $\Lambda(\mathbf{O})$.

We can say still more. Let $\left\{\mathscr{B}_{p}\right\}_{p \geqslant 1}$ denote any of the following families of varieties: $\left\{\mathbf{P}_{2 p+1,0}\right\},\left\{\mathbf{P}_{2 p, 0}\right\},\left\{\mathbf{M S}^{(p)}\right\}$. Then, when ordered as a subset of the join-irreducible elements of $\Lambda_{f}(\mathbf{O}),\left\{\mathscr{B}_{p}\right\}_{p \geq 1}$ contains a copy of $\{1,2, \ldots\}$ ordered by divisibility. This ordered set in turn contains a copy of $2^{k}$ for every $k \geqslant 1$. Thus all the equivalence classes we have considered are, in a strong sense, 'large'.

5. Other Classes of Relative Ockham Lattices. We begin with an extremely elementary result, but one which contrasts sharply with those in Section 3.

Proposition 5.1. Let $\mathscr{A} \in \Lambda(\mathbf{O})$ and suppose $\mathscr{A} \supseteq \mathbf{P}_{2,1}$. Then every finite distributive lattice is a relative $\mathscr{A}$-lattice.

Proof. Let $P$ be any non-empty finite ordered set. Fix any point $y$ of $P$ and define $g$ by $g(x)=y$ for all $x \in P$. Clearly $g^{2}=g$, so $P$ has been made into the dual of an algebra in $\mathbf{P}_{2,1}$.

Proposition 5.1 implies that every finite distributive lattice is a relative $\mathbf{P}_{m, n}$-lattice whenever $m>n \geqslant 1$ (cf. Propositions 4.4 and 4.6).

We observe that in general the dual $X$ of a finite subdirectly irreducible Ockham algebra is such that $X^{\mathbf{P}_{2.1}} \in \mathrm{GM}(X)$ if and only if the end of $X$ is an order component. Algebraically this means that $\mathscr{A} \in \Lambda_{f}(\mathbf{0})$ contains $\mathbf{P}_{2,1}$ if and only if some finite subdirectly algebra in $\mathscr{A}$ has 2 as direct factor.

We take up in a later paper the question of whether the Ockham varieties $\mathscr{A}$ for which $\mathscr{L}^{\mathscr{A}}$ is the class of all finite distributive lattices are precisely those containing $\mathbf{P}_{2,1}$.

Describing the lattice of subvarieties of $\mathbf{P}_{2,1}$ is entirely straightforward using Theorem 4.1 (see M. S. Goldberg [15]). The lattice shown in Fig. 5 is obtained.

In view of the contrast between Proposition 5.1 and Theorem 3.7 we are now immediately led to seek $\mathscr{L}^{S \vee S^{d}}$. It turns out that the variety $\mathbf{S} \vee \mathbf{S}^{d}$ nicely illustrates the sort of order-theoretic arguments that can arise when one considers other Ockham varieties, and helps to put the results of Section 3 into perspective. 


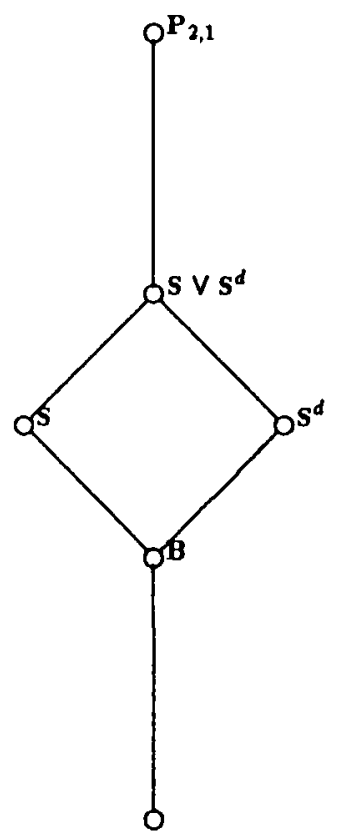

Figure 5

Equationally, the variety $\mathbf{S} \vee \mathbf{S}^{d}$ is given, as a subvariety of $\mathbf{P}_{2,1}$, by

$$
a \wedge \sim a \leqslant b \vee \sim b .
$$

This identity holds in an Ockham algebra $A$ if and only if every point $x$ of the dual space of $A$ is comparable to its $g$-image $g(x)$ (see [1]).

LEMma 5.2. A non-empty finite ordered set $P$ can be made into the dual space of an algebra in $\mathbf{S} \vee \mathbf{S}^{d}$ if and only if it is the disjoint union of sets of the form $\downarrow x \cup \uparrow x(x \in P)$, that is, if and only if every order-component of $P$ has a node.

Proof. On a set

$$
P=\bigcup_{1 \leqslant i \leqslant k} \downarrow x_{i} \cup \uparrow x_{i}
$$

a $g$-map may be defined by $g(x)=x_{i}$, whenever $x$ is comparable to $x_{i}$. Then $g$ is order-reversing, $g^{2}=g$, and each point is comparable to its $g$-image.

Conversely, suppose $(P ; g)$ is the dual space of a finite algebra in $\mathbf{S} \vee \mathbf{S}^{d}$. Let $Z=\{g(x): x \in P\}$. Then $Z$ is an antichain (since $g=g^{2}$ ). Because each point is comparable with its $g$-image, $P=\downarrow Z \cup \uparrow Z$. It follows that $P$ is of the desired form.

We now need to characterise those finite ordered sets all of whose non-empty convex subsets are as in Lemma 5.2. We do this in two stages. First we prove a lemma which shows that no ordered set of the kind we require can contain $\mathbf{N}$ as a subposet, and then we use Lemma 3.2 to identify the appropriate subclass of ordered sets of this type.

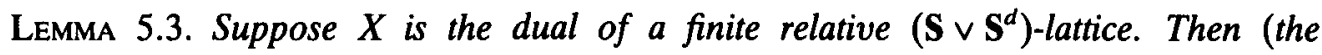
underlying ordered set of) $X$ has no subposet isomorphic to $\mathbf{N}$. 

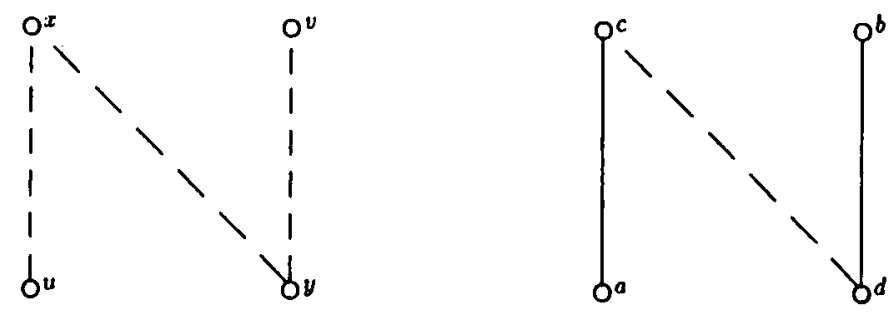

Figure 6

Proof. Suppose $X$ has $\mathbf{N}$ as a subposet, so there exist elements $\{u, v, x, y\}$ such that $u<x, x>y, y<v, u\|v, u\| y$ and $x \| v$.

We claim that $X$ has as a convex subposet an ordered set $Q$ of the following form. There exist points $\{a, b, c, d\}$ such that $Q=\{a, b\} \cup[d, c], a \dashv c, b \succ d, d<c, a \| b$, $a \| d$ and $c \| b$. To find such a subposet we first consider

$$
X_{1}=\uparrow u \cap \uparrow y \cap(X \backslash \uparrow v)
$$

This set contains $x$, so is non-empty. If it contained an element below $v$, then $u \leqslant v$, contrary to hypothesis. Take $x_{1}$ to be a minimal element of $X_{1}$. Then $\left\{u, x_{1}, y, v\right\}$ is still a subposet isomorphic to $\mathbf{N}$. Now consider $X_{2}=\downarrow x_{1} \cap(X \backslash \uparrow y) \cap(X \backslash \downarrow y) \cap \uparrow u ; X_{2}$ contains $u$, so is non-empty. Choose $u_{1}$ to be a maximal element of $X_{2}$. If $u_{1} \leqslant v$, then $u \leqslant v$, a contradiction, and if we had $u_{1} \geqslant v$, we would have $x_{1} \geqslant v$, which is also impossible. By construction, $u_{1} \| y$. We claim $u_{1} \prec x_{1}$. Suppose there were a point $u_{2}$ such that $u_{1}<u_{2}<x_{1}$. We have $u \leqslant u_{1}<u_{2}$, so $u_{2} \in \uparrow u$. If $u_{2} \in \uparrow v$, then $x_{1} \geqslant \uparrow v$, which is false. We already know $u_{2} \notin \uparrow y, u_{2} \in \downarrow x_{1}$, and $u_{2} \geqslant u$. Finally, if $u_{2} \in \downarrow y$, then $u_{1} \in \downarrow y$. These observations show that $u_{2}$ lies in $X_{2}$ and we have a contradiction to the maximality of $u_{1}$. Carrying out the same process dually on the other arm of $\mathbf{N}$, we arrive at the required set $Q$.

Take a subposet $Q=\{a, b, c, d\}$ in $X$ forming an $\mathbf{N}$ as above, so $Q=\{a, b\} \cup[d, c]$, $a \prec c, b \succ d, d<c, a\|b, a\| d$ and $c \| b$. Suppose (for contradiction) that $g$ could be defined on $Q$ to make it the dual of an algebra in $\mathbf{S} \vee \mathbf{S}^{d}$. Necessarily $g(a)=a$ or $g(a)=c$. Suppose the former. Then $g(c)=a$ and $g(d) \geqslant g(c)$, so that $g(d)=a$ or $c$. Also (because of the comparability condition on $g), g(d)$ is $d$ or $b$, so we are forced to have $g(d)=a$ and $g(b)=d$. But then $g(b) \neq g^{2}(b)$. The same argument rules out $g(b)=b$. Now assume $g(a)=c$ and $g(b)=d$. The condition $g^{2}=g$ entails $g(c)=c$ and $g(d)=d$, and we see that $g$ cannot be order-reversing. (Alternatively, we may appeal to Lemma 5.2.) to $\mathbf{N}$.

We shall denote by $N$ the distributive lattice shown in Fig. 7; it is the lattice dual

The finite distributive lattices which fail to have $N$ as a homomorphic image are exactly those whose duals fail to contain $\mathbf{N}$ as a subposet, while those which do not have $N$ as an interval are those whose duals are $\mathbf{N}$-free, that is, fail to have $\mathbf{N}$ as a convex subposet. Both these classes of ordered sets play an important role in order theory, as shown by M. Habib and R. H. Möhring [17] and I. Rival [21].

As Lemma 5.3 indicates, it is the class of ordered sets not containing $\mathbf{N}$ as a subposet which is of relevance to our investigation of relative $\left(\mathbf{S} \vee \mathbf{S}^{d}\right)$-lattices. A result independently discovered by many authors shows that this class coincides with the class of 


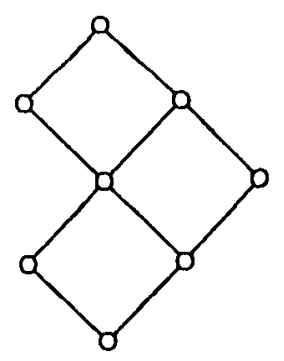

Figure 7

series-parallel posets (see [17] and [21] for references and further characterisations). A finite ordered set is said to be series-parallel if it is the empty set or can be constructed from singleton sets using the operations of disjoint sum and linear sum. We thus have that the dual spaces of algebras in $\mathscr{L}^{\mathbf{S} \vee \mathbf{S}^{d}}$ are series-parallel posets. It is easy to see that not all series-parallel posets arise this way: Lemma 5.2, or the same sort of argument used in the last part of the proof of Lemma 5.3, shows that the four element ordered set, $\bowtie$, shown in Fig. 8 cannot be made into an $\left(\mathbf{S} \vee \mathbf{S}^{d}\right)$ dual space.

Clearly, $\bowtie$ is series-parallel-it is the linear sum of two 2-element antichains. It turns out that the exclusion of $\bowtie$ is exactly what we need.

Proposition 5.4. Suppose that $P$ is a non-empty finite series-parallel poset. Then the following are equivalent:

(1) $P$ is such that every order-component has a node;

(2) $P$ does not have $\bowtie$ as a convex subposet;

(3) in the construction of $P$ from singletons, the following are permitted: $(i)$ disjoint union, (ii) linear sum, with the proviso that the formation of $P_{1} \oplus P_{2}$ is permitted only when $P_{1}$ has a largest element or $P_{2}$ has a smallest element, or both.

Proof. We have already observed that (1) implies (2). To show (2) implies (3), we argue by contradiction. If (3) fails, then at some stage of the series-parallel construction of $P$, a linear sum $P_{1} \oplus P_{2}$ is formed in which $\left|\max P_{1}\right| \geqslant 2$ and $\left|\min P_{2}\right| \geqslant 2$. We can then form $\bowtie$ as a convex subposet of $P_{1} \oplus P_{2}$ using two maximal points of $P_{1}$ and two minimal points of $P_{2}$. Now note that convexity is not destroyed by later stages of the series-parallel construction.

Finally we show that (3) implies (1). Suppose we consider finite series-parallel posets built up under the restriction imposed in (3). We prove by structural induction that every

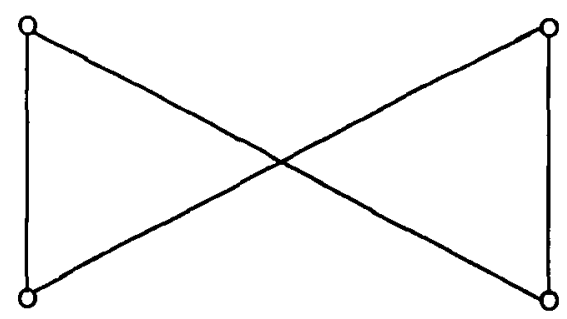

Figure 8 
such ordered set is such that every order-component has a node. Suppose that $P$ is a finite series-parallel poset such that its substructures have this property. Let $Q$ be any convex subposet of $P$. There are two cases. If $P$ is the disjoint union of substructures $P_{1}$ and $P_{2}$, then $Q \cap P_{1}$ and $Q \cap P_{2}$ are convex subposets of $P_{1}$ and $P_{2}$ respectively, and every order-component of each has a node, by hypothesis. In the other case, $P$ is the linear sum $P_{1} \oplus P_{2}$ of substructures $P_{1}$ and $P_{2}$. If $Q \subseteq P_{1}$ or $Q \subseteq P_{2}$, we can apply our inductive hypothesis. Otherwise, $Q \cap P_{1} \neq \emptyset$ and $Q \cap P_{2} \neq \emptyset$. Then the largest element of $P_{1}$ or the smallest element of $P_{2}$, whichever exists, provides the required node for $Q$ (which in this case is necessarily connected).

We remark that the convexity restriction cannot be removed from condition (2) in Proposition 5.4. Consider $\mathrm{X}$, the ordered set with underlying set $\{a, b, c, d, x\}$ and order given by $a \prec x \prec b, c \prec x \prec d$. The subset obtained by removing $x$ is isomorphic to $\bowtie$.

Theorem 5.5 translates Proposition 5.4 into algebraic terms. Given finite lattices $L_{1}$ and $L_{2}$, we denote their reduced linear sum by $L_{1} \oplus L_{2}$; this is obtained by taking the linear sum of $L_{1}$ and $L_{2}$ and identifying the top element of $L_{1}$ with the bottom element of $L_{2}$. The non-trivial finite distributive lattices whose duals are series-parallel posets are those which can be built up from 2-element chains using direct product and reduced linear sum.

THEOREM 5.5. Let $L$ be a finite distributive lattice. Then the following are equivalent.

(1) $L \in \mathscr{L}^{\mathbf{S} \vee \mathbf{S}^{d}}$,

(2) $L$ is a member of the class $\mathscr{C}$ defined as follows:

(a) the trivial algebra and the two element chain are in $\mathscr{C}$,

(b) the direct product of two members of $\mathscr{C}$ is in $\mathscr{C}$,

(c) if $L_{1}$ and $L_{2}$ are in $\mathscr{C} \cup\{\emptyset\}$, then $L_{1} \oplus 1 \oplus L_{2}$ is in $\mathscr{C}$,

(d) every member of $\mathscr{C}$ is obtained in a finite number of steps using (a), (b), (c).

(3) $L$ does not have $N$ as a homomorphic image and $L$ contains no subinterval isomorphic to $2^{2} \bar{\oplus} 2^{2}$.

Criterion (2) fits neatly with the constructive descriptions in Theorem 3.7 of finite relative Stone and relative dual Stone lattices. For the varieties $\mathscr{A}$ considered in Section $3, \mathscr{L}^{\star}$ could be described either by excluded subintervals or by excluded quotients. However, already for $\mathbf{S} \vee \mathbf{S}^{d}$, condition (3) in Theorem 5.5 suggests that we must abandon the hope that finite relative Ockham lattices can generally be characterised via a set of

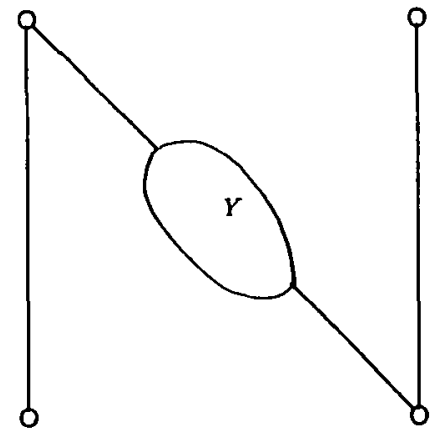

Figure 9 
excluded subintervals. This is confirmed by the following argument. Suppose that $\mathscr{L}^{S \vee \mathbf{S}^{d}}$ could be characterised by the exclusion of certain lattices as subintervals. Then there would be a class $\mathscr{L}$ of ordered sets such that the duals of algebras in $\mathscr{L}^{\mathbf{S} \vee \mathbf{S}^{d}}$ would be precisely those finite ordered sets which fail to contain any member of $\mathscr{Z}$ as a convex subposet. Now consider the ordered set $P$ depicted in Figure 9.

Here $Y$ represents the underlying ordered set of any non-trivial algebra in $\mathscr{L}^{\mathbf{S} \vee \mathbf{S}^{d}}$. This set $P$ is not the dual of a relative $\left(S \vee S^{d}\right)$-lattice. Its non-empty proper convex subsets either are convex subsets of $Y$ or have a maximum or a minimum element. Clearly none of these can lie in $\mathscr{Z}$, since all can be made into $\left(\mathbf{S} \vee \mathbf{S}^{d}\right)$ dual spaces. Therefore $P$ itself must be in $\mathscr{Z}$. Thus not only could $\mathscr{Z}$ not be finite but it would of necessity contain a family of sets in one-to-one correspondence with the sets whose characterisation is being sought. This circularity shows that no worthwhile 'excluded subintervals' result can exist in this case.

We remark in conclusion that the same sort of situation occurs elsewhere. We have regarded the variety $\mathbf{S}$ as a variety of Ockham algebras. It can also be considered as a subvariety of the variety $\mathbf{B}_{\omega}$ of pseudocomplemented distributive lattices. It is the variety $\mathbf{B}_{1}$ in the chain of subvarieties

$$
\mathbf{B}_{-1} \subset \mathbf{B}_{0} \subset \mathbf{B}_{1}=\mathbf{S} \subset \mathbf{B}_{2} \subset \ldots \subset \mathbf{B}_{\omega}
$$

of $\mathbf{B}_{\omega}$. (See, for example, [2] for details and background.) The variety $\mathbf{B}_{n}$ consists of those algebras $A$ in $\mathbf{B}_{\omega}$ which are such that each prime ideal of $A$ contains at most $n$ minimal prime ideals. The following result, noted independently by W. H. Cornish [10] and B. A. Davey [11], is the natural generalisation of Theorem 1.2, condition (2): a finite distributive lattice is a relative $\mathbf{B}_{n}$-lattice if and only if it does not have $2^{n+1} \oplus \mathbf{1}$ as a homomorphic image. Equivalently, a finite distributive lattice is a relative $\mathbf{B}_{n}$-lattice if and only if its dual space does not contain as a subposet $\overline{n+1} \oplus 1$, where $\bar{k}$ denotes a $k$-element antichain.

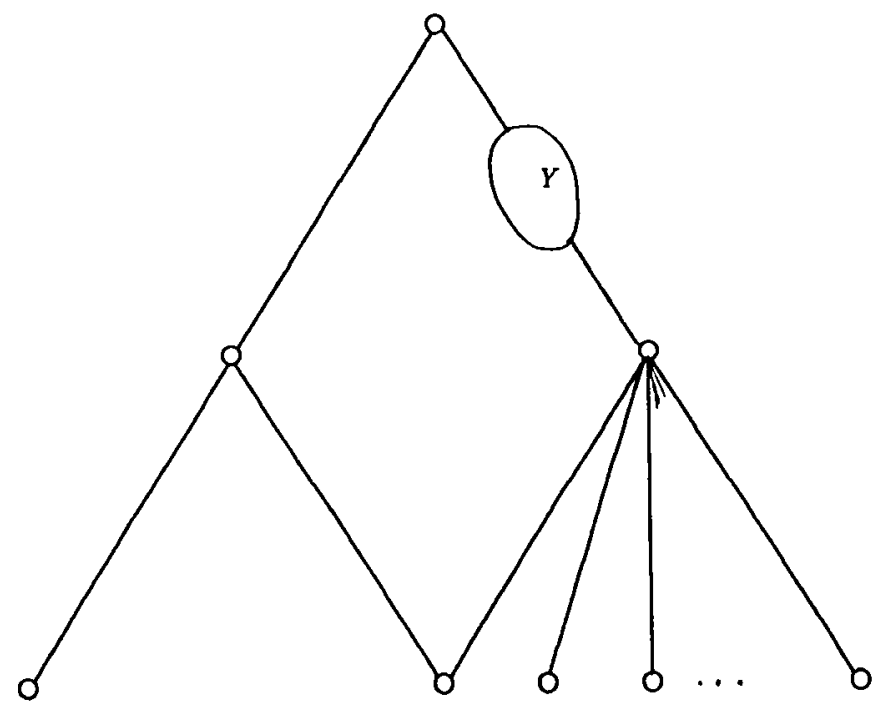

Figure 10 
The fundamental reason that excluded subintervals can be used to describe $\mathscr{L}^{\mathscr{A}}$, for $\mathscr{A}=\mathbf{B}, \mathbf{K}, \mathbf{S}$, and $\mathbf{S}^{d}$, is that each of $\mathbf{I}, \mathbf{V}$ and $\Lambda$ has the highly special property that it occurs as a convex subposet of a finite ordered set if it occurs at all. This property fails to extend to $\overline{n+1} \oplus 1$ for $n \geqslant 2$, and it is a straightforward matter to show that, for $n \geqslant 2$, no useful characterisation of finite relative $\mathbf{B}_{n}$-lattices by excluded subintervals is possible. One argues in the same way as for $\mathbf{S} \vee \mathbf{S}^{d}$, using ordered sets of the form shown in Figure 10 , where $Y$ is any ordered set dual to a relative $\mathbf{B}_{n}$-lattice.

\section{REFERENCES}

1. M. E. Adams and H. A. Priestley, Equational bases for varieties of Ockham algebras, Preprint.

2. R. Balbes and Ph. Dwinger, Distributive lattices (University of Missouri Press, 1974).

3. J. Berman, Distributive lattices with an additional unary operation, Aequationes Math. 16 (1977), 165-171.

4. A. Björner, On complements in lattices of finite length, Discrete Math. 36 (1981), 325-326.

5. T. S. Blyth, A. S. A. Noor and J. C. Varlet, Ockham algebras with de Morgan skeleton, $J$. Algebra 117 (1988), 165-178.

6. T. S. Blyth and J. C. Varlet, On a common abstraction of de Morgan algebras and Stone algebras, Proc. Roy. Soc. Edinburgh Sect. A 94 (1983), 301-308.

7. T. S. Blyth and J. C. Varlet, Subvarieties of the class of MS-algebras, Proc. Roy. Soc. Edinburgh Sect. A 95 (1983), 157-169.

8. T. S. Blyth and J. C. Varlet, MS-algebras definable on a distributive lattice, Bull. Roy. Soc. Liège 54 (1985), 167-182. (to appear).

9. G. Bordalo, A duality between unary algebras and their subuniverse lattices, Port. Math.

10. W. H. Cornish, $n$-normal lattices, Proc. Amer. Math. Soc. 45 (1974), $48-53$.

11. B. A. Davey, Some annihilator conditions on distributive lattices, Algebra Universalis 4 (1974), 316-322.

12. B. A. Davey, On the lattice of subvarieties, Houston J. Math. 5 (1979), 183-192.

13. B. A. Davey and D. Duffus, Exponentiation and duality, in "Ordered sets," NATO Advanced Study Institutes Series, Reidel Publ. Co. (1982), 43-95.

14. B. A. Davey and $H$. Werner, Dualities and equivalences for varieties of algebras, Colloq. Math. Soc. Janos Bolyai 33 (1983), 101-275.

15. M. S. Goldberg, Distributive p-algebras and Ockham algebras, Ph.D. Thesis, La Trobe University, Bundoora, Australia (1979).

16. M. S. Goldberg, Distributive Ockham algebras: free algebras and injectivity, Bull. Austral. Math. Soc. 24 (1981), 161-203.

17. M. Habib and R. H. Möhring, On some complexity properties of $N$-free posets and posets with bounded decomposition diameter, Discrete Math. 63 (1987), 157-182.

18. B. Jónsson, Algebras whose congruence lattices are distributive, Math. Scand. 21 (1967), $110-121$.

19. H. A. Priestley, Ordered sets and duality for distributive lattices, Ann. Discrete Math. 23 (1984), 39-60.

20. M. Ramalho and M. Sequeira, On generalised MS-algebras, Port. Math. 44 (1987), 315-328.

21. I. Rival, Stories about the letter N, Contemporary Math. 57 (1986), 263-285.

22. A. Urquhart, Lattices with a dual homomorphic operation, Studia Logia 38 (1979), 201-209. 
23. A. Urquhart, Lattices with a dual homomorphic operation II, Studia Logia 40 (1981), 391-404.

24. J. Varlet, Relative de Morgan lattices, Discrete Math. 46 (1983), 207-209.

Faculdade de Ciencias,

Departmento de Matematica, Rua ERnesto de Vasconcelos, BLoco C1, PIso $3_{-}^{\circ}$, 1700 Lisboa, Portugal.
Mathematical Instrtute, 24-29 ST. GiLES, OXFORD OX1 3LB, ENGLAND. 